\title{
Lock Acquisition \& Commissioning of the Advanced Virgo Detector
}

\section{Diego Bersanetti*}

University of Genoa \& INFN Genoa

E-mail: diego.bersanetti@ge.infn.it

Gravitational waves are ripples of the space-time metric caused by astrophysical events, as predicted by Einstein's Theory of General Relativity. The recent publication, by the LIGO-Virgo Collaboration, of the first detection of such waves shed new light upon this method of investigation.

The Advanced Virgo experiment will join in 2017 the international network of interferometric detectors, after the end of the commissioning. A crucial aspect of the commissioning is the development of a lock acquisition strategy, which is the procedure that brings the optically resonant cavities of the interferometer in their working point, using advanced control schemes. The main features of such procedure for this 2 nd generation detector will be presented, with a focus on the ongoing commissioning.

XVII International Workshop on Neutrino Telescopes

13-17 March 2017

Venezia, Italy

${ }^{*}$ Speaker. 


\section{Introduction}

Advanced Virgo [1] is an interferometric gravitational wave detector, whose optical layout is depicted in Fig. 1; its detection technique is to measure the phase difference between the laser light travelling through the two perpendicular arms of a Michelson interferometer.

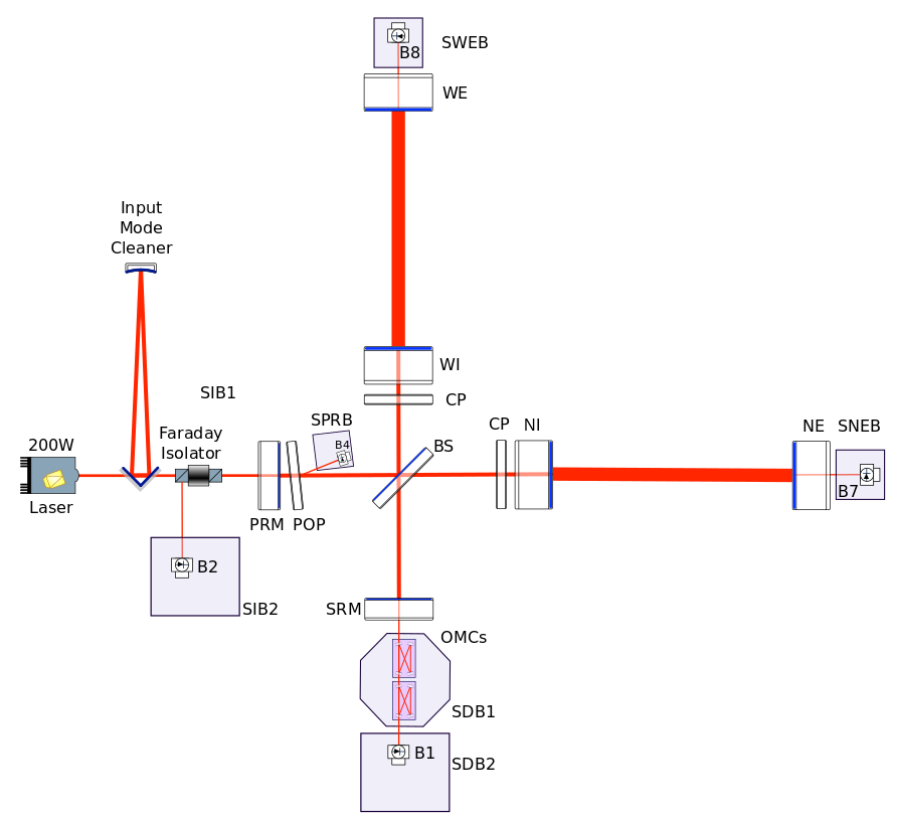

Credit: The Virgo Collaboration

Figure 1: Optical layout of Advanced Virgo.

Its basic sensitivity is enhanced by substituting the arms with resonant Fabry-Pérot cavities, which keep the light trapped in the interferometer, increasing the effective arms' length which translates into a higher sensitivity; another improvement is provided by the Power Recycling mirror, placed before the beam splitter, which allows to re-inject the light that would be lost at the symmetric port, thus increasing the circulating power and, therefore, the sensitivity.

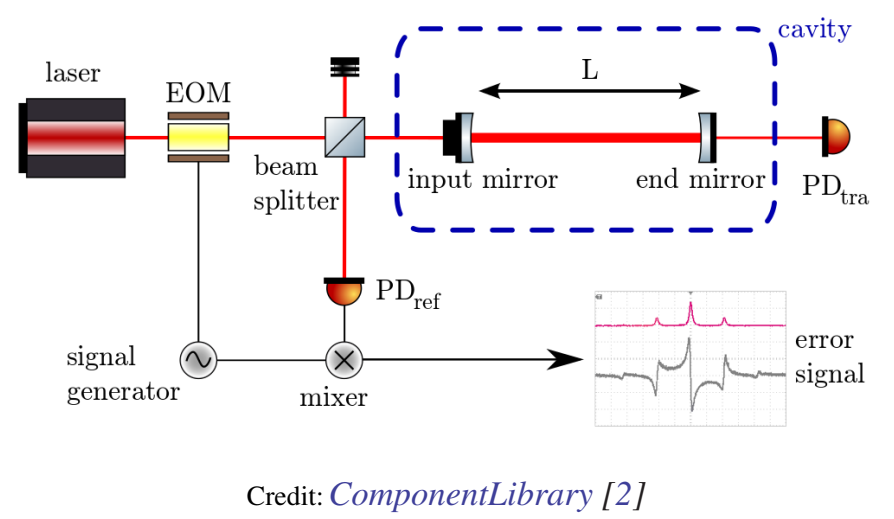

Figure 2: Pound-Drever-Hall locking scheme for a Fabry-Pérot cavity. 
In order to work properly, all optical cavities need to be locked, which means that their length must be very accurately controlled. This is done using the Pound-Drever-Hall [3, 4] technique, which uses electro-optical modulation to generate a radio-frequency error signal which can be used in a linear feedback control loop, as shown in Fig. 2 for an arm cavity.

\section{Lock Acquisition of Advanced Virgo}

A very important part of the Commissioning of the detector is the development of the Lock Acquisition sequence, which is a sequential series of steps where all the optical cavities and their several degrees of freedom are brought to their optically resonant working point.

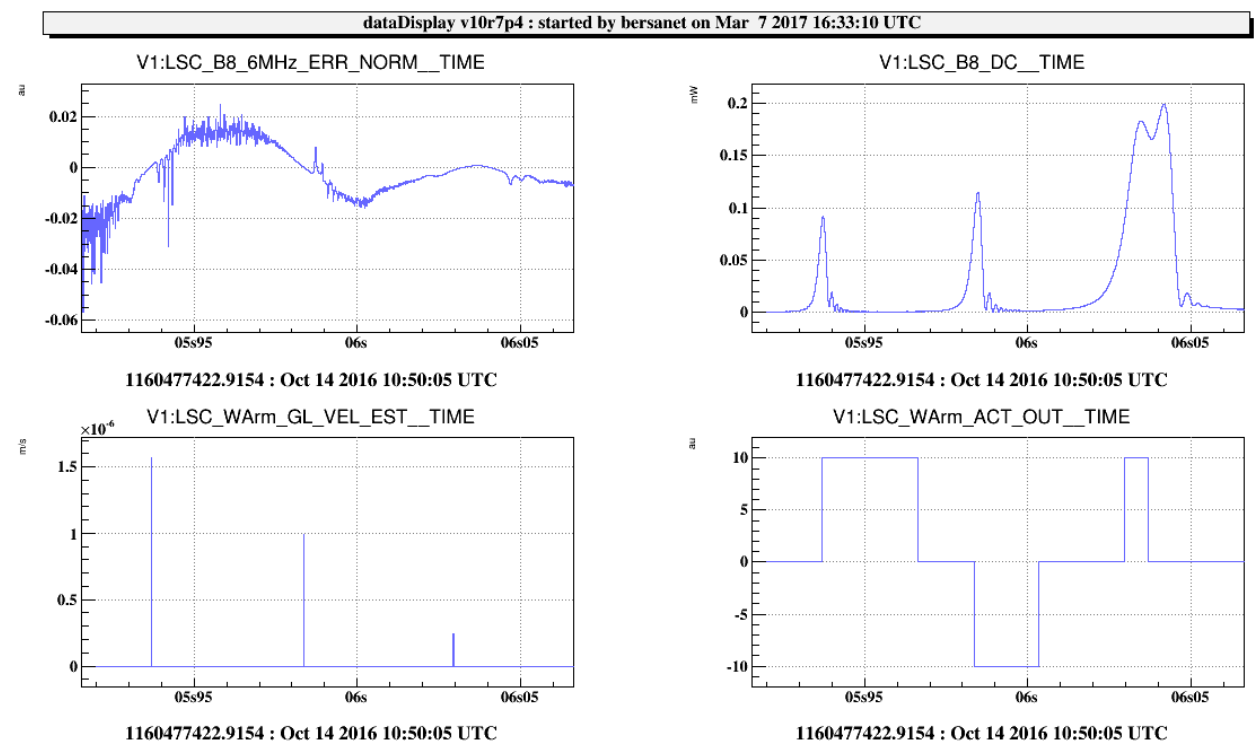

Figure 3: Application of the Guided Lock [5, 6] algorithm, used to lock an arm cavity of Advanced Virgo.

For Advanced Virgo, this sequence starts with the lock of the arm cavities, using the Guided Lock [5, 6] algorithm to estimate the relative velocity of a cavity (Fig. 3, bottom-left panel) and then, if necessary, slow it down with calibrated impulses (Fig. 3, bottom-right panel) in order to use a Pound-Drever-Hall error signal (Fig. 3, top-left panel) to lock the cavity and bring the power (Fig. 3, top-right panel) to its resonance condition. After the independent arm cavities are locked, their controlled lengths are geometrically converted into common and differential arm motion, allowing to suppress the former with the frequency stabilization of the main laser, and to use the latter as the gravitational wave detection signal.

Afterwards, the sequence proceeds with locking the Short Michelson degree of freedom, and eventually the Power Recycling cavity's length, using an updated version of the Variable Finesse Technique [7] which was already used for the 1st Generation configurations (Virgo and, later, Virgo+): the interferometer's working point is eventually reached by balancing the central Michelson interferometer, which means to bring the length difference between the arms of the short Michelson towards zero ("MICH offset reduction", as shown in the bottom-right panel of Fig. 4); this allows to reach the "Dark Fringe" condition, where the light going towards the detec- 
tion port of the interferometer is virtually zero, allowing a more sensitive detection of an incoming gravitational wave signal.

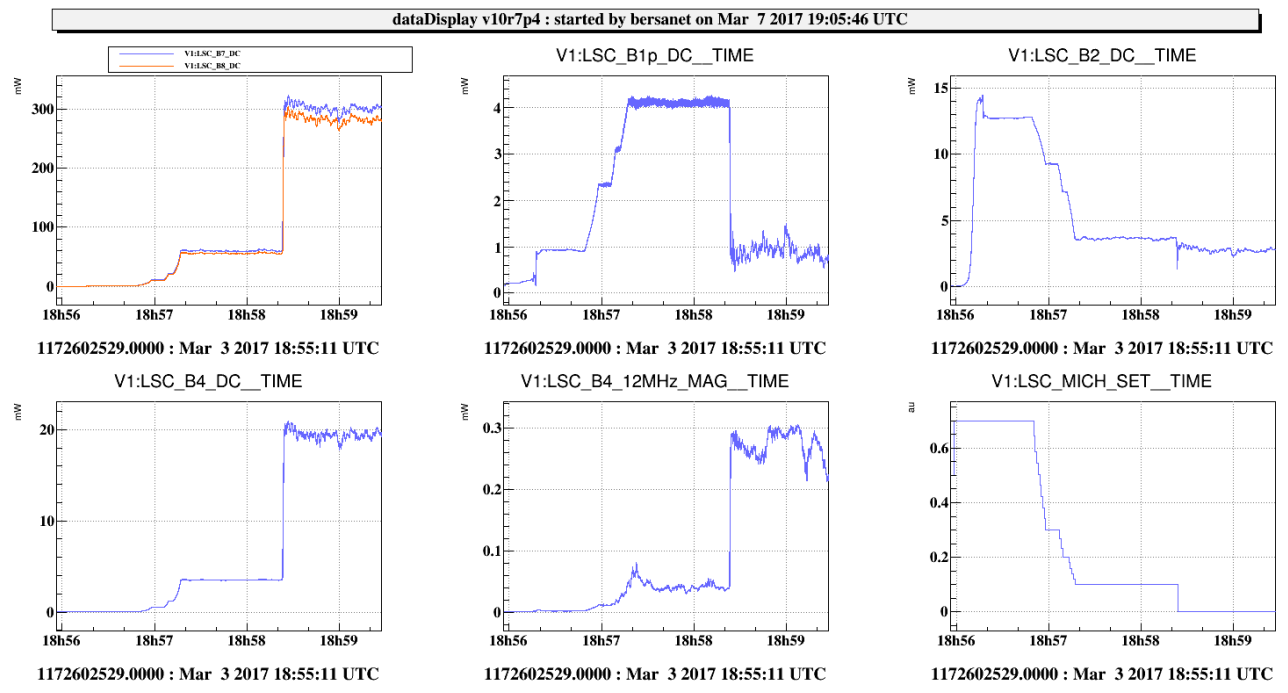

Figure 4: Lock Acquisition procedure for Advanced Virgo. Top row, left to right: transmitted cavity powers, asymmetric port power, symmetric port power. Bottom row, left to right: Power-Recycling Cavity ("PRC") power, PRC sidebands' power, rescaled Michelson's unbalance, from 1 ("bright fringe") to 0 ("dark fringe").

The Commissioning of the Advanced Virgo detector will conclude in the first half of 2017, and it will be immediately followed by noise hunting activities, in order to increase the sensitivity of the apparatus and improve the portion of observable universe; at the end of this phase, the detector will join the two LIGO interferometers for the last part of the $O 2$ Observational Run, when for the first time three 2nd Generation gravitational wave interferometric detectors will be taking data jointly and will push forward the gravitational wave astronomy, born in 2016 with the announcement of the first gravitational wave signal ever observed, the GW150914 event [8].

\section{References}

[1] F. Acernese et al., Class. Quantum Grav. 32, 024001 (2015)

[2] A. Franzen, ComponentLibrary (a vector graphics library for illustrations of optics experiments), http://www.gwoptics.org/ComponentLibrary/, Licensed under a Creative Commons Attribution-NonCommercial 3.0 Unported License

[3] R. W. P. Drever et al., Appl. Phys. B, 31, 97 (1983)

[4] E. D. Black, American Journal of Physics 69, 79 (2001)

[5] J. Camp et al., Opt. Lett. 20, 2463 (1995)

[6] D. Bersanetti, Development of a New Lock Acquisition Strategy for the Arm Cavities of Advanced Virgo, Ph.D. Thesis, Università degli Studi di Genova (Genova, Italy) (2016)

[7] L. Barsotti, The Control of the Virgo Interferometer for Gravitational Waves Detection, Ph.D. Thesis, Università degli Studi di Pisa (Pisa, Italy) (2006)

[8] B. P. Abbott et al., Phys. Rev. Lett. 116, 061102 